\title{
Ovarian vein thrombosis after delivery
}

\author{
Signe Østergaard'; Anne-Mette Hvas²; Lise Medrud ${ }^{3}$; Jens Fuglsang ${ }^{1}$ \\ ${ }^{1}$ Department of Obstetrics and Gynaecology, Aarhus University Hospital; ${ }^{2}$ Department of Clinical Biochemistry , Aar- \\ hus University Hospital; ${ }^{3}$ Department of Radiology , Aarhus University Hospital
}

Ovarian vein thrombosis (OVT) is reported to be an infrequent complication after delivery with a prevalence of $0.02-0.2 \%$ of all pregnancies (1). Risk factors may include caesarean section (CS), twin delivery, and infection (1). Sepsis, propagating thrombosis and pulmonary embolism are potential complications, why a high case-fatality rate has been ascribed to OVTs $(1,2)$.

Case 1: A 32-year-old woman had a vaginal delivery at term, manual removal of the placenta, concomitant $2100 \mathrm{ml}$ bleeding and transfusion of two portions of red blood cells.

Recovery was uneventful with early hospital discharge. On day four the patient was readmitted with fever, pain in the lower left abdomen, leucocytosis and increased C-reactive protein. Endometritis was suspected. Symptoms persisted despite antibiotics. On day eight she started having trouble lifting her left leg. A computer tomography (CT) scan of the abdomen demonstrated an OVT at the left side ( $>$ Figure 1. CT scan; coronal view. The arrow points to the OVT on the left side). Treatment with Tinzaparin (175 IU/kg) was instituted. Three months of anticoagulant therapy with Warfarin followed. No further readmission took place. A subsequent thrombophilia screening was normal.

Case 2: A 35-year-old twin pregnant woman had an acute CS in week $35+4$ as the water had broken. Total bleeding was

Phlebologie 2018; 47: 277-278

https://doi.org/10.12687/phleb2446-5-2018

Nachdruck aus und zu zitieren als:

Hämostaseologie 2018; 47: 9-10

https://doi.org/10.5482/HAM0-17-09-0032

received: September 22, 2017

accepted in revised form: January 17, 2018
$1050 \mathrm{ml}$. Hypertension and acute fatty liver of pregnancy developed. Biochemical parameters normalised, but physical recovery was slow. Due to immobilisation prophylactic dose of Tinzaparin was commenced. On day eight the patient had pain and a palpable swelling in the lower right abdomen. The D-dimer level increased to $16.0 \mathrm{mg} / \mathrm{L}$ (ref. interval $<0.5 \mathrm{mg} / \mathrm{L}$ ). Antithrombin levels and clotting times were normal. A CT scan demonstrated a $10 \times 7 \times 14 \mathrm{~cm}$ haematoma in the rectus muscle. Additionally, bilateral OVT was found ( Figure 2a. CT scan; Coronal view. The arrows point to the left and the right OVT).

Prophylactic, not therapeutic, doses of Tinzaparin were chosen (4,500 IU daily). A repeated CT scan after 16 days demonstrated re-canalisation of the ovarian veins $(\triangleright$ Figure 2b. CT scan; Coronal view. The arrow points to the recanalization of the OVT on the left side). The haematoma vanished and no other complications were noted. A normal thrombophilia screening followed.

The two cases presented show the complexity of diagnosing OVT.

Diagnosing OVT incidentally has been reported recently, and OVT has been proposed to be a frequent or even a physiological event after delivery (3-5). Less severe cases could mimic common disease entities after delivery, e.g. endometritis or urinary tract infections. Self-limitating symptoms may be observed in case of spontaneous recanalization. The diagnosis rely on a CT scan or magnetic resonance imaging.

No guidelines for the treatment of OVT are currently available. Here, anticoagulant regimens were different due to the presence of a large haematoma in case \#2. In pregnancy-related cases anticoagulation may be optional, but three months of anticoagulation has been considered safe $(3,5)$. We observed re-canalisation of the ovarian vein after 16 days of prophylactic low molecular weight heparin treatment.

We suggest that a diagnosis of OVT should be followed by three months of anticoagulation in therapeutic doses. Thrombophilia screening may in some cases be relevant. However, individual approaches may be necessary.

\section{Conflicts of interest}

None to be declared.

\section{Compliance with Ethical Standard}

Informed consent was obtained from all individual participants included in the study.

\section{References}

1. Angelini M, Barillari G, Londero AP, Bertozzi S, Bernardi S, Petri R et al. Puerperal ovarian vein thrombosis: two case reports. J Thromb Thrombolysis 2013; 35(2): 286-289.

2. Hippach M, Meyberg R, Villena-Heinsen C, Mink D, Ertan AK, Schmidt W et al. Postpartum ovarian vein thrombosis. Clin Exp Obstet Gynecol 2000; 27(1): 24-26.

3. Plastini T, Henry D, Dunleavy K. Ovarian vein thrombus: To treat or not to treat? Blood Advances 2017; 1(15): 1120-1123.

4. Khalil H, Avruch L, Olivier A, Walker M, Rodger $\mathrm{M}$. The natural history of pelvic vein thrombosis on magnetic resonance venography after vaginal delivery. Am J Obstet Gynecol 2012; 206(4): 356.e1-4.

5. Rottenstreich A, Da'as N, Kleinstern G, Spectre G, Amsalem H, Kalish Y. Pregnancy and non-pregnancy related ovarian vein thrombosis: Clinical course and outcome. Thromb Res 2016; 146: 84-88. 


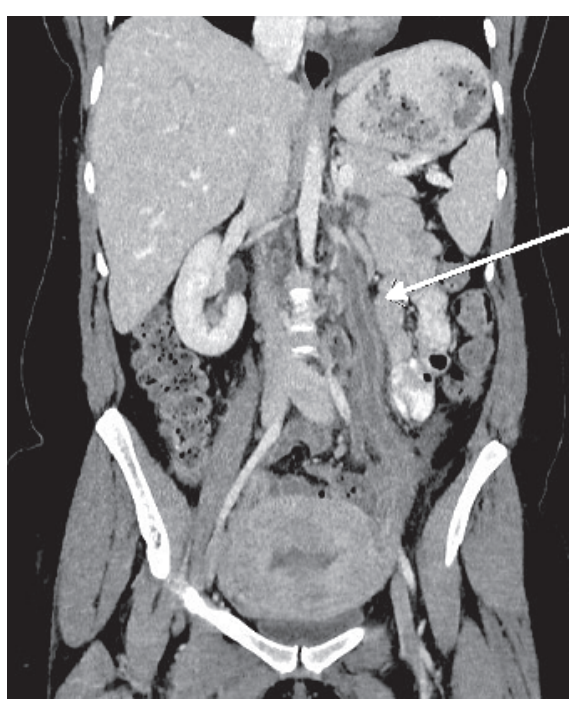

Fig. 1

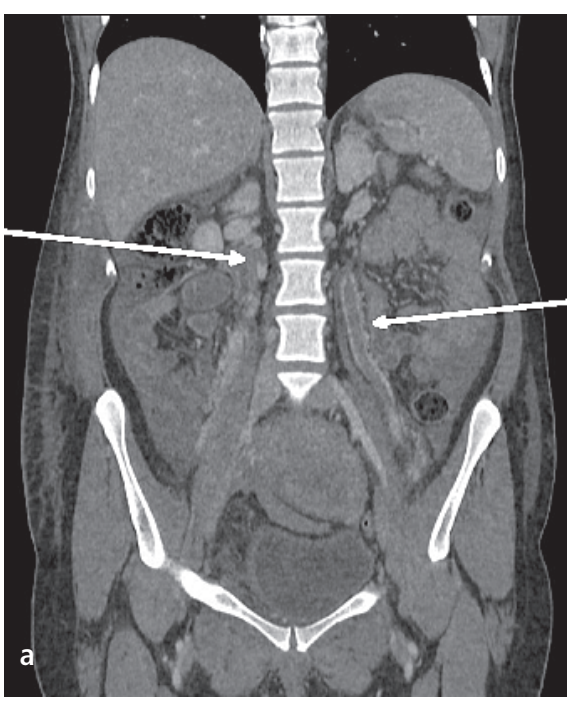

Fig. 2

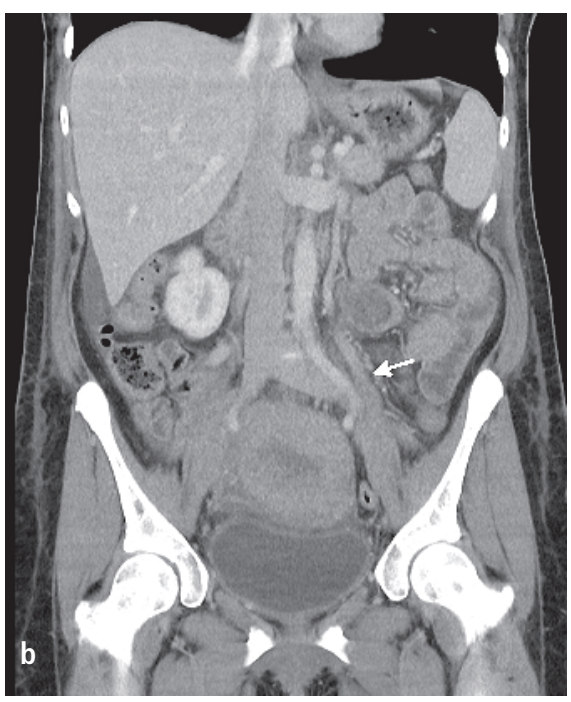

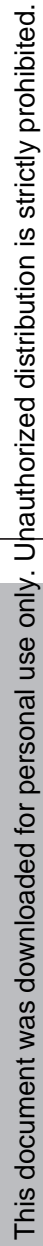

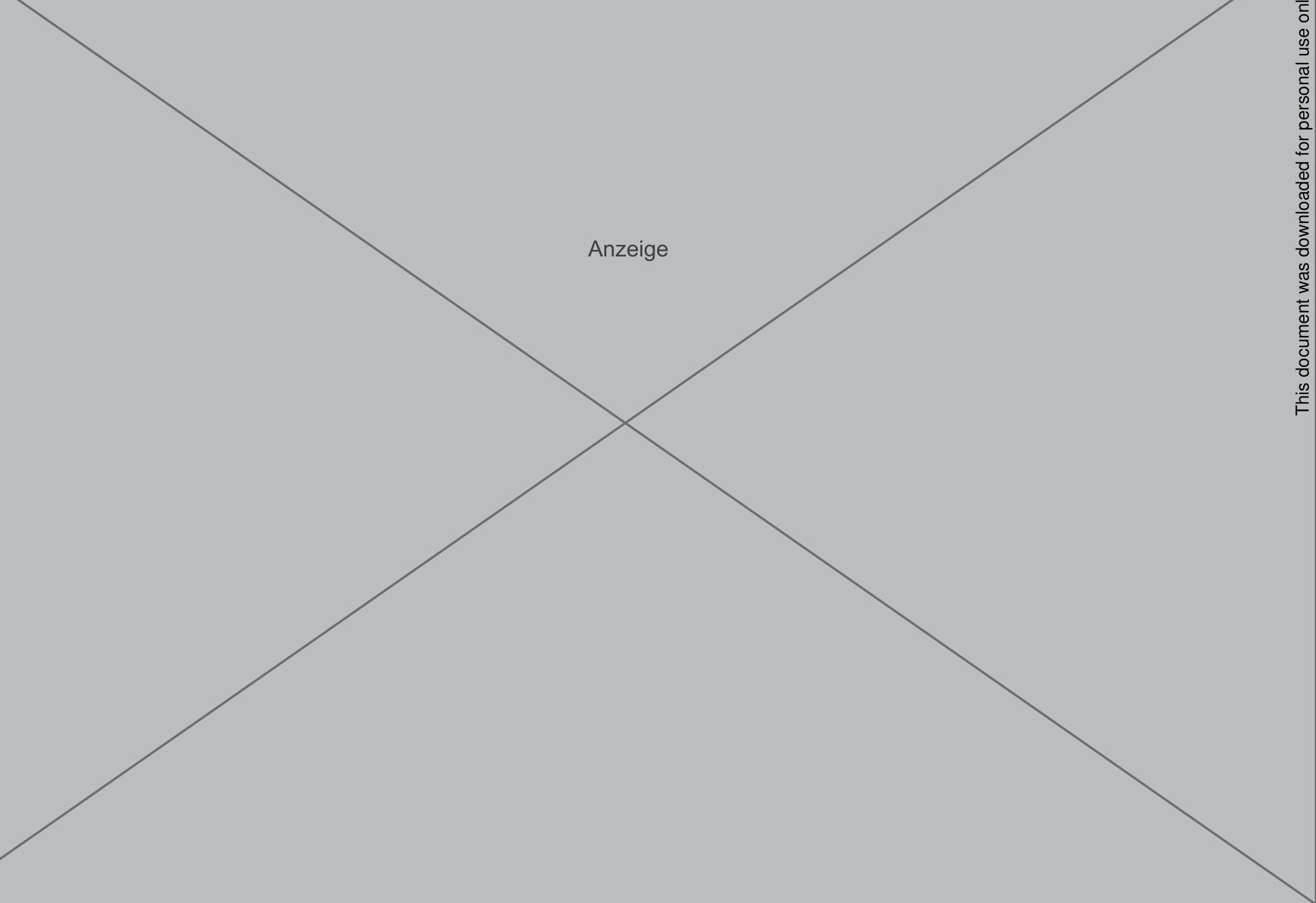

